\title{
Explanation of the embedding strength of particle board
}

\author{
T.A.C.M. van der Put
}

Published online: 27 March 2008

(C) The Author(s) 2008

\begin{abstract}
By the earlier derived theory of the embedding strength, based on the equilibrium method of plasticity, it is possible to fully explain the empirical relations and test results of an investigation of the embedding strength of particle boards leading to a new insight for the right design rules.
\end{abstract}

\section{Erklärung der Lochleibungsfestigkeit von Spanplatten}

Zusammenfassung Anhand der bereits früher auf Grundlage des Traglastverfahrens hergeleiteten Theorie zur Lochleibungsfestigkeit können die Versuchsergebnisse und empirischen Gleichungen einer Untersuchung zur Lochleibungsfestigkeit von Spanplatten vollständig erklärt werden. Dies führt zu neuen Erkenntnissen im Hinblick auf richtige Berechnungsregeln.

\section{Introduction}

For the design of pin dowel joints it is necessary to know the embedding strength in different situations. In Budianto et al. (1977), test results are given of an extended investigation of the embedding strength of structural particle boards. In the following, the, for applications always necessary theoretical explanation is given of these test results. The theory was developed in 1979 in the research reports of the TU-Delft (Reports hsc-3 to 6) and applied in the Dutch investigations and regulations and is extended in van der Put (2006). Contrary to the empirical equations of Budianto et al. (1977), based

\section{T.A.C.M. van der Put (区)}

Faculty of Civil Engineering and Geosciences,

Timber structures and wood technology, TU Delft,

P.O. Box 5048, 2600 GA Delft, The Netherlands

e-mail: vanderp@xs4all.nl on the dowel slenderness, the $a / d$ ratio of Fig. 3, the theory shows the strength to be dependent on the spreading width, the $b / d$ ratio, according to Eq. 9. This is verified in Dutch investigations and shown here by the test-results at constant $a / d$ with different $b / a$ ratios by using e.g. one plate thickness and one dowel diameter with different $b / d$ ratios. This is lacking in Budianto et al. (1977) and therefore also in the Code rules.

It thus is necessary to adapt the Codes at these points for the right design.

\section{Influence of the density on the strength}

If the random oriented and evenly distributed pores are modeled to a spherical volume $V_{p}$ with radius $r$, given in Fig. 1, in agreement with the mean pore volume of an unit volume of the material at the determining spot, then the mean strength $\sigma$ due to flow of the material at the smallest cross section is:

$\sigma=\frac{1-\pi r^{2}}{1} \cdot \sigma_{0}$,

where $\sigma_{0}$ is the strength of the material.

Fig. 1 Unit volume Abb. 1 Porenvolumen

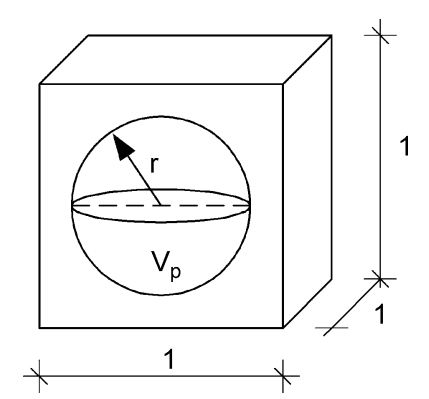


With the pore volume:

$V_{p}=4 \pi r^{3} / 3$,

the mean density is:

$\rho=\left(1-V_{p}\right) \rho_{0} \quad$ or: $\quad V_{p}=1-\rho / \rho_{0}$

where $\rho_{0}$ is the density of the material. Thus Eq. 1 can be written:

$\sigma=\sigma_{0}\left(1-\pi\left(\frac{3}{4 \pi} V_{p}\right)^{2 / 3}\right)=\sigma_{0}\left(1-1.2\left(V_{p}\right)^{2 / 3}\right)$,

$\sigma=\sigma_{0}\left(1-1.2\left(1-\rho / \rho_{0}\right)^{2 / 3}\right)$.

Because $\rho \ll \rho_{0}$, this can be approximated to:

$\sigma \approx \sigma_{0}\left(1-1.2\left(1-2 / 3 \cdot \rho / \rho_{0}\right)\right)=-0.2 \sigma_{0}+0.8 \sigma_{0}\left(\rho / \rho_{0}\right)$

according to the first term of the row-expansion.

Equation 5 thus has the form of

$\sigma=c_{1} \rho-c_{2}$

and $c_{1} / c_{2}=0.8 \sigma_{0} /\left(0.2 \sigma_{0} \rho_{0}\right)=4 / \rho_{0}$.

The density of the cell wall is about $1.6 \mathrm{~g} / \mathrm{cm}^{3}$. This wall also has pores and filling material and a better value of $\rho_{0}$ of the bearing material is 1.7 to 1.8 or with $\rho_{0}=1.75 \mathrm{~g} / \mathrm{cm}^{3}$, it is

$c_{1} / c_{2}=4 / 1.75=2.3$.

This is in agreement with the empirical lines of Fig. 16 of Budianto et al. (1977) given in Fig. 2. For the different pindiameters " $d$ " the measured lines, Eq. 5, are:

$d=2 \mathrm{~mm}: \quad \sigma=482 \rho-261 c_{1} / c_{2}=(1.9)$

$d=4 \mathrm{~mm}: \quad \sigma=148 \rho-53 \quad c_{1} / c_{2}=2.7$

$d=6 \mathrm{~mm}: \quad \sigma=167 \rho-80 \quad c_{1} / c_{2}=2.1$

$d=8 \mathrm{~mm}: \quad \sigma=137 \rho-65 \quad c_{1} / c_{2}=2.1$

$d=10 \mathrm{~mm}: \quad \sigma=109 \rho-48 \quad c_{1} / c_{2}=2.3$.

Mean value: $c_{1} / c_{2}=2.3$.

It can be seen in Fig. 2 that the spreading around the $d=2 \mathrm{~mm}$ line is high and this causes an empirical value of $c_{1} / c_{2}=1.9$ instead of 2.3. Calculation of $\sigma_{0}$ from $c_{1}$ and $c_{2}$ of Eqs. 5 and 6 shows that the slope of the $d=4 \mathrm{~mm}$ line is also different. Because of the high spreading, the theoretical slopes according to $c_{1} / c_{2}=2.3$ will be as probable as the empirical ones of Fig. 2.

The density correction of the strength at the same dowel diameter thus is:

$\frac{\sigma_{1}}{\sigma_{2}}=\frac{2.3 \rho_{1}-1}{2.3 \rho_{2}-1}$.

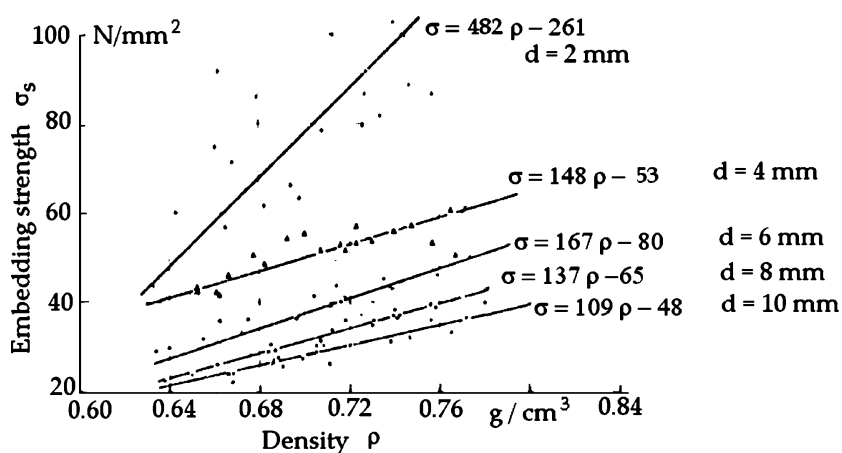

Fig. 2 Embedding strength dependent on the density; $a=19 \mathrm{~mm}$ Abb. 2 Lochleibungsfestigkeit in Abhängigkeit der Rohdichte; $a=$ $19 \mathrm{~mm}$

Fig. 3 Cross section with pin dowel loading Abb. 3 Querschnitt mit Stabdübelbelastung

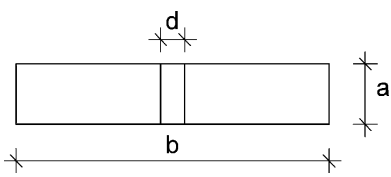

The strength $\sigma_{0}$ shows to decrease with the increase of the pin diameter as will be explained in the next paragraphs by the spreading effect and volume effect.

\section{Influence of the spreading possibility of the pin-dowel loading}

The spreading of the stresses below the local loading by the pin causes confined dilatation and thus a high embedding strength.

It can be derived from the theory of plasticity, discussed in van der Put (2006), that the embedding strength $\sigma_{s}$ can safely be given by:

$\sigma_{s}=\sigma_{c} \cdot \sqrt{\frac{b a}{d a}}=\sigma_{c} \cdot \sqrt{\frac{b}{d}} \quad\left(\leq 4.7 \cdot \sigma_{c}\right)$,

where " $a$ " and " $b$ " are the dimensions of the cross section of an embedding test specimen, given in Fig. 3, " $d$ " the diameter of the dowel and where $\sigma_{c}$ is the compression strength because for $d=b$ the compression strength is reached, (that should be measured on the specimen for $d=b$ ). The strength difference, due to the spreading effect alone, for specimens with the same dimensions but different diameters " $d$ " of the pin-dowels is:

$\frac{\sigma_{1}}{\sigma_{2}} \approx \sqrt{\frac{d_{2} b}{d_{1} b}}=\left(\frac{d_{2}}{d_{1}}\right)^{0.5}$

and it follows that the strength decreases with the increase of the pin diameter.

Besides this spreading effect there is the influence of a volume effect for small diameters " $d$ " as discussed in the next paragraph. 


\section{Combined spreading- and volume effect of the embedding strength}

Splitting of the material below the pin-dowel is necessary for high plastic deformation and the initial flow strength depends on the weakest section and decreases with the increase of the volume according to:

$\sigma=\sigma_{1}\left(\frac{V_{1}}{V}\right)^{1 / k}$,

where $\sigma_{1}$ is the mean strength for the specimen with stressed volume $V_{1}$ and $\mathrm{k}$ is dependent on the coefficient of variation $S / \sigma$ according to:

$\left(\frac{S}{\sigma}\right)^{2}=\frac{\Gamma(1+2 / k)}{\Gamma^{2}(1+1 / k)}-1$.

From the row expansion of the Gamma functions it can be seen that approximately holds:

$\frac{S}{\sigma}=\frac{c}{k} \approx \frac{1.2}{k}$,

where $c$ is a little varying function of $S / \sigma$ in the usual range of $S / \sigma$ and is approximately constant with a value of $c \approx 1.2$. For an ideal fracture by the weakest plane, $S / \sigma$ remains constant or: $S / \sigma=S_{1} / \sigma_{1}$ and Eq. 11 becomes:

$\sigma=\sigma_{1}\left(\frac{V_{1}}{V}\right)^{S_{1} / 1.2 \sigma_{1}}=\sigma_{1}\left(\frac{V_{1}}{V}\right)^{0.83 v_{1}}$.

The strength difference, due to the combined spreading effect and the volume effect, for specimens with the same dimensions but different small diameters of the pin-dowels is:

$\frac{\sigma_{1}}{\sigma_{2}} \approx\left(\frac{d_{2}}{d_{1}}\right)^{(0.5+0.83 \cdot 0.2)}=\left(\frac{d_{2}}{d_{1}}\right)^{0.66}$,

where it is assumed that the coefficient of variation $v_{1}$ of Eq. 14 is 0.2 .

In Fig. 17 of Budianto et al. (1977), given here as Fig. 4, test results are given on a particle board plate of $a=19 \mathrm{~mm}$, following the empirical relation:

$\sigma_{s}=7.85 \cdot(a / d)+13.91$

for $a / d$ values between 2 and 6 . Above $a / d=6$ the curve bends down and is horizontal at $a / d \approx 9$ to 10 . The strength ratio according to Eq. 16 is:

$\frac{\sigma_{1}}{\sigma_{2}}=\frac{7.85 \cdot a / d_{1}+13.91}{7.85 \cdot a / d_{2}+13.91}$.

For these test at constant " $a$ ", Eq. 15 also can be expressed in $a / d$ as follows:

$\frac{\sigma_{1}}{\sigma_{2}}=\left(\frac{d_{2}}{d_{1}}\right)^{0.66}=\left(\frac{a / d_{1}}{a / d_{2}}\right)^{0.66}$.

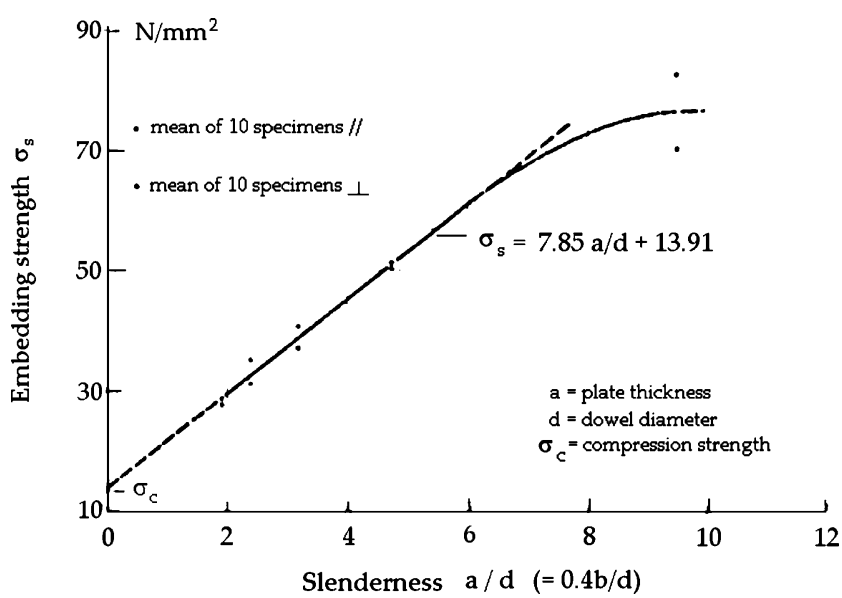

Fig. 4 Embedding strength dependent on $a / d ; a=19 \mathrm{~mm}$

Abb. 4 Lochleibungsfestigkeit in Abhängigkeit des Schlankheitsgra$\operatorname{des} a / d ; a=19 \mathrm{~mm}$

Taking $a / d_{2}=2$ as reference, then for $a / d_{1}=6$, is $\sigma_{1} / \sigma_{2}=$ $(6 / 2)^{0.66}=2.06$. The same follows from the empirical Eq. 17: $\sigma_{1} / \sigma_{2}=2.06$. For $a / d_{1}=4$, Eq. 18 gives a ratio $\sigma_{1} / \sigma_{2}=(4 / 2)^{0.66}=1.58$, while this is 1.53 according to Eq. 17. At high values of $a / d$ the line bends down (see Fig. 4). At $a / d_{1} \approx 9$, where the line is about horizontal, Eq. 18 predicts a strength of:

$$
\begin{aligned}
\sigma_{1} & =\left(\frac{a / d_{1}}{a / d_{2}}\right)^{0.66} \cdot \sigma_{2}=\left(\frac{9}{2}\right)^{0.66} \cdot \sigma_{2}=2.7 \cdot \sigma_{2} \\
& =2.7 \cdot 29.6=80 \mathrm{MPa}
\end{aligned}
$$

while measured it is about $76 \mathrm{MPa}$. There thus is a limit given by a horizontal line. The horizontal line means that failure is independent of the dimensions $a / d$ of the specimen and a local failure mechanism around the pin-dowel is determining because of the large dimension with respect to the dowel $(a / d=9$ and $b / d=2.5 \cdot 9=21.5)$. For specimens with a higher $b / d$ than $b / d \approx 22$ thus $b / d=22$ has to be substituted in Eq. 9. The high spreading of the data around the mean value of this mechanism is due to the strong influence of the manufacturing direction of the plate on the strength as is shown in Budianto et al. (1977).

It now can be concluded that the theoretical expression, Eq. 18 is fully able to explain the test results, and as well the straight part as the curved part of the line of Fig. 4.

\section{Approximate equations for the embedding strength}

To be able to explain the empirical approximate relations of the data of Budianto et al. (1977), a linearization of Eqs. 9 and 11 is necessary. The embedding strength with respect to a reference diameter $d_{0}$ can be written according to Eqs. 9 
and 15:

$\sigma_{s}=\sigma_{c} \cdot\left(\frac{d_{0}}{d}\right)^{0.16} \cdot \sqrt{\frac{b}{d}}=\sigma_{c} \cdot\left(\frac{d_{0}}{d}\right)^{0.16} \cdot \sqrt{\frac{2.5 \cdot a}{d}}$,

because of standard dimension of the specimen, chosen in the investigation of Budianto et al. (1977), of $b \approx 2.5 \cdot a$, Eq. 19 can be written:

$$
\begin{aligned}
\sigma_{s}= & \sigma_{c} \cdot\left(c_{3}-c_{3}+\frac{d_{0}}{d}\right)^{0.16} \cdot\left(c_{4}-c_{4}+\frac{2.5 \cdot a}{d}\right)^{0.5} \text { or: } \\
\sigma_{s}= & \sigma_{c} \cdot\left(c_{3}\right)^{0.16} \cdot\left(c_{4}\right)^{0.5} \cdot\left(1+\frac{d_{0} / d-c_{3}}{c_{3}}\right)^{0.16} \\
& \cdot\left(1+\frac{2.5 \cdot a / d-c_{4}}{c_{4}}\right)^{0.5} \\
\approx & \sigma_{c} \cdot\left(c_{3}\right)^{0.16} \cdot\left(c_{4}\right)^{0.5} \cdot\left(1+0.16 \cdot \frac{d_{0} / d-c_{3}}{c_{3}}\right) \\
& \cdot\left(1+0.5 \cdot \frac{2.5 \cdot a / d-c_{4}}{c_{4}}\right),
\end{aligned}
$$

according to the first term of a row expansion. This is allowed when sufficient high values of $c_{3}$ and $c_{4}$ are chosen to satisfy: $\left(d_{0} / d-c_{3}\right) / c_{3} \ll 1$ and $\left(2.5 a / d-c_{4}\right) / c_{4} \ll 1$. Thus:

$$
\begin{aligned}
\sigma_{s} \approx & \sigma_{c} \cdot\left(c_{3}\right)^{0.16} \cdot\left(c_{4}\right)^{0.5} \cdot\left(0.84+0.16 \cdot \frac{d_{0}}{c_{3} d}\right) \\
& \cdot\left(0.5+0.5 \cdot \frac{2.5 \cdot a}{c_{4} d}\right) \\
= & 0.42 \cdot \sigma_{c} \cdot\left(c_{3}\right)^{0.16} \cdot\left(c_{4}\right)^{0.5} \cdot\left(1+0.19 \cdot \frac{d_{0}}{c_{3} d}\right) \\
& \cdot\left(1+\frac{2.5 \cdot a}{c_{4} d}\right) \\
\approx & 0.42 \cdot \sigma_{c} \cdot\left(c_{3}\right)^{0.16} \cdot\left(c_{4}\right)^{0.5} \cdot\left(1+0.19 \cdot \frac{d_{0}}{c_{3} d}+\frac{2.5 \cdot a}{c_{4} d}\right) .
\end{aligned}
$$

This last step is a further approximation to obtain an equation that is identical to the empirical equation of Budianto et al. (1977):

$\sigma_{s}=\sigma_{c}+\frac{4.3 \cdot a+69}{d}$.

This means that: $0.42 \cdot\left(c_{3}\right)^{0.16} \cdot\left(c_{4}\right)^{0.5} \approx 1 ; c_{4}=2.5 \sigma_{c} / 4.3=$ $0.58 \sigma_{c}$ and $c_{3}=0.19 \sigma_{c} d_{0} / 69=0.00275 \cdot \sigma_{c} d_{0}$. For the particle board plate of Fig. 4 is $\sigma_{c}=13.9 \mathrm{MPa}$ giving $d_{0} \approx$ $9 \mathrm{~mm}$.

It can be seen that Eq. 22 does not apply in general. The empirical constants 4.3 and 69 are proportional to the mean compression strength of all types of plates of the investigation instead of being proportional to the own compression strength of each type of plate. Further the slope of the $\sigma_{s}-$ $a / d$ line is not constant equal to 4.3 , but increases with the decrease of $\mathrm{d}$ as follows from the factor $\left(1+0.19 \cdot d_{0} / c_{3} d\right)$

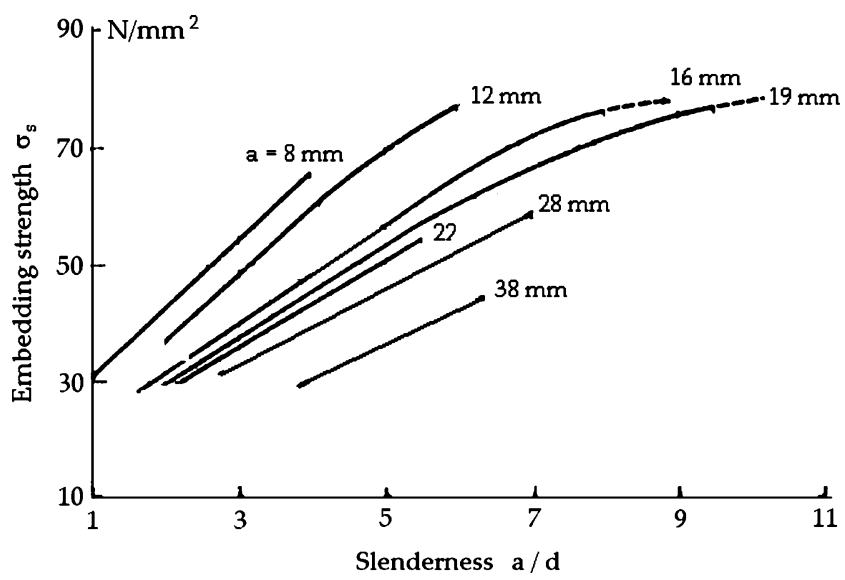

Fig. 5 Embedding strength dependent on $a / d$ with $a=8$ to $38 \mathrm{~mm}$ Abb. 5 Lochleibungsfestigkeit in Abhängigkeit des Schlankheitsgrades $a / d$ mit $a=8$ bis $38 \mathrm{~mm}$

of Eq. 20 and can be seen in Fig. 5 (being Fig. 18 of Budianto et al. (1977)). But the main reason is that the linearization of the volume effect is not right because $\left(d_{0} / d-\right.$ $\left.c_{3}\right) / c_{3} \ll 1$ is not satisfied. Thus Eq. 19 should be approximated to:

$\sigma_{s} \approx 0.5 \cdot \sigma_{c} \cdot\left(c_{4}\right)^{0.5}\left(\frac{d_{0}}{d}\right)^{0.16} \cdot\left(1+\frac{2.5 \cdot a}{c_{4} d}\right)$.

This line is fitted to go through $\sigma_{c}$ so that $c_{4}$ is chosen to follow:

$0.5 \cdot\left(c_{4}\right)^{0.5}\left(\frac{d_{0}}{d}\right)^{0.16}=1 \quad$ or: $\quad c_{4}=4 /\left(d_{0} / d\right)^{0.32}$

and the slope of the line Eq. 23 is:

$$
\begin{aligned}
\frac{\partial \sigma_{s}}{\partial(a / d)} & =0.5 \cdot \sigma_{c} \cdot\left(c_{4}\right)^{0.5}\left(\frac{d_{0}}{d}\right)^{0.16} \cdot \frac{2.5}{c_{4}} \\
& =0.62 \cdot \sigma_{c} \cdot\left(\frac{d_{0}}{d}\right)^{0.32} \cdot
\end{aligned}
$$

Thus for $d_{1}=38 \mathrm{~mm}$ and $d_{2}=8 \mathrm{~mm}$, the extremes of Fig. 5, the slopes ratio is:

$\frac{\left(\partial \sigma_{s} / \partial(a / d)\right)_{1}}{\left(\partial \sigma_{s} / \partial(a / d)\right)_{2}}=\left(\frac{d_{2}}{d_{1}}\right)^{0.32}=\left(\frac{8}{38}\right)^{0.32}=0.6$.

The measured ratio of the slopes in Fig. 5 is about 0.5 , indicating that the power should be 0.44 instead of 0.32 , thus that the coefficient of variation of the overall spreading is: $v_{1}=0.22 \cdot 1.2=0.26$ in stead of 0.20 as is for the separate test series of Fig. 4. The reason of the higher spreading is the mean compression strength of all series (and not of each series) that is regarded and probably not for all series $b / a$ is the same. Then " $a$ " can not replace " $b$ " in Eq. 19 as is done. (The dimensions of the specimens are not given in detail in Budianto et al. (1977).) 
The volume effect of Eq. 14 does not apply for larger bearing plates of local loads because the splitting effect occurs at the plate edge and this volume at the edge is the same for different plate dimensions " $d$ ". Thus for $d>d_{0}, d=d_{0}$ has to be inserted in the equations and then Eq. 9 applies. A similar equation, Eq. 27, applies for the Dutch tests with one constant diameter " $d$ " (for the same volume effect in all tests) at different $a / d$ and $b / d$ values:

$\sigma_{s}=c_{5} \cdot \sigma_{c} \cdot \sqrt{\frac{b}{d}}$.

This is shown to be right in many cases (see e.g. the TUDelft Reports HSC 3 to 6). Then, the constant $c_{5}$ is an empirical constant by the volume effect and the testing effect, when the compression strength is not measured under the same test conditions on a specimen with other dimensions than " $a$ " times " $b$ " of the embedding strength specimen.

\section{Experimental verification of the embedding strength theory}

In order to verify the theory of the embedding strength, it was necessary to vary $b / d$. This has not been regarded in Budianto et al. (1977). Furthermore, the influence of other boundary conditions had to be tested.

In Fig. 6, the form of the specimen is given as used for the investigation of Budianto et al. (1977). Because of the high embedding strength it was necessary to stiffen the pin-dowel and a slit was used instead of a round hole.

It also appeared that for $a / d>3$ to 4 , the difference with a round hole was negligible if there was sufficient material around the slit. An open slit gives a different deformation behaviour. Because of the expected minor influence of the boundary conditions at the dowel on the strength, other specimen forms where also tested in the Dutch investigation. For instance, the simple specimen with dimensions $a-b-e$

Fig. 6 Embedding strength specimen

Abb. 6 Probekörper zur Prüfung der Lochleibungsfestigkeit

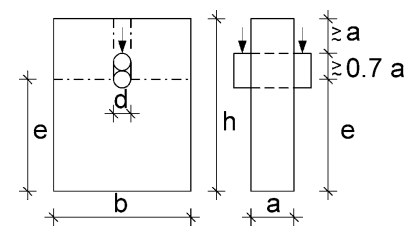

Fig. 7 Pin push test Abb. 7 Dübeleindrückversuch (see Fig. 6), with a half round hole (open slit) was chosen and also a specimen without a slit (Fig. 7).

In the investigation of Budianto et al. (1977), $e / d>8$ to 10 was chosen to obtain sufficient plastic deformation and to avoid splitting: $b>\sim 2.5 \cdot a$. However splitting is always active, even at local embedding failure, and determines the ultimate value. So this restriction was not followed in the Dutch investigation and to show that the spreading length " $b$ " and not " $a$ " has an influence on the strength, also specimens with smaller " $b$ " than " $a$ " values were tested. For the variation of $b / d$ and $a / d, e=b=10 d$ and $b=5 d$ with $d_{1}=a / 4$ and $d_{2}=a / 8$ were chosen in the pin push tests. These tests of pushing a pin into the specimen according to Fig. 7 were done by TNO Delft. Only a deformation of $1 \cdot d$ is possible in these tests. Flow occurred for $d_{1}=5 \mathrm{~mm}$. This was not the case for $d_{2}=2.5 \mathrm{~mm}$, due to the higher strength by the volume effect. Thus the hardening to the spreading mechanism is not always measurable with this type of test. This hardening effect increases with $b / d$ and flow at maximal spreading occurs after larger deformations.

The start of flow $\sigma_{s}^{\prime}$ also follows Eq. 27:

$\sigma_{s}^{\prime}=c \cdot \sigma_{c} \cdot \sqrt{b / d}$

where $\sigma_{c}$ is the compression strength; $c \approx 0.95$ for $d=$ $5 \mathrm{~mm}$ and $b / d=4$, with $\sigma_{c}=24.9 \mathrm{~N} / \mathrm{mm}^{2}$, coefficient of variation 0.05 for a sample size of 5 specimens. $\sigma_{c}^{\prime}=$ $47.5 \mathrm{~N} / \mathrm{mm}^{2}$, coefficient of variation 0.03 for a sample size of four specimens. The specimen dimensions were: $a=$ $18 \mathrm{~mm} ; b=20 \mathrm{~mm}$ and $60 \mathrm{~mm}$ heights. The testing time was 30 to $60 \mathrm{sec}$.

In the main investigation of the Stevin-laboratory, tests were done with higher plastic deformations. Three types of specimens are used as given in Fig. 6 with $b / d=5$ and $b / d=10$ with open and closed slits. The dimensions in $\mathrm{mm}$ of the specimen with closed slit (series A) were: $h=100$; $e=b=67 ; a=22 ; d=7$ of the slit and of the steel dowel $d=6.7 \mathrm{~mm}$. The dimensions in $\mathrm{mm}$ of the specimens with open half round slit were: $h=e=b=67$ (series B) and $h=e=67$ with $b=33.5$, (series $\mathrm{C}$ ) both series with $d=7$ and $a=22$. The 3 series $\mathrm{A}, \mathrm{B}$, and $\mathrm{C}$ had the same $a / d$ ratio. A board type with relatively low splitting strength and thus a low tensile strength perpendicular to the plate of $0.27 \mathrm{~N} / \mathrm{mm}^{2}$ was chosen. The compression strength in plate direction was $10.9 \mathrm{~N} / \mathrm{mm}^{2}$ at a density of $0.615 \mathrm{~g} / \mathrm{cm}^{3}$ (determined by TNO). The specimens of each series were taken besides each other from the board, giving differences in the densities between the series. Thus the embedding strength was corrected according to Eq. 8:

$\frac{\sigma_{1}}{\sigma_{2}}=\frac{2.3 \cdot \rho_{1}-1}{2.3 \cdot \rho_{2}-1}$.

The expected embedding strength according to: $\sigma_{s}=\sigma_{c}$. $\sqrt{b / d}$ is: 
- For series A with $b / d=10, \rho=0.71 \mathrm{~g} / \mathrm{cm}^{3}$ and closed slit:

$\sigma_{s}=10.9 \cdot \frac{2.3 \cdot 0.71-1}{2.3 \cdot 0.615-1} \cdot \sqrt{10}=52.6 \mathrm{~N} / \mathrm{mm}^{2}$.

Measured was $53.9 \mathrm{~N} / \mathrm{mm}^{2}$, c.o.v. 0.06 for a sample of 5 specimens.

- For series B with $b / d=10, \rho=0.7 \mathrm{~g} / \mathrm{cm}^{3}$ and half round open slit:

$\sigma_{s}=52.6 \cdot \frac{2.3 \cdot 0.7-1}{2.3 \cdot 0.71-1}=50.7 \mathrm{~N} / \mathrm{mm}^{2}$.

Measured was $49.4 \mathrm{~N} / \mathrm{mm}^{2}$, c.o.v. 0.11 for a sample of 6 specimens.

- For series $\mathrm{C}$ with $b / d=5, \rho=0.63 \mathrm{~g} / \mathrm{cm}^{3}$ and half round open slit:

$\sigma_{s}=10.9 \cdot \frac{2.3 \cdot 0.63-1}{2.3 \cdot 0.615-1} \cdot \sqrt{5}=26.4 \mathrm{~N} / \mathrm{mm}^{2}$.

Measured was $27.3 \mathrm{~N} / \mathrm{mm}^{2}$, c.o.v. 0.11 for a sample of 6 specimens.

Thus the open slit is as strong as the closed slit for all cases and the value of $c$ of Eq. 27 is $c=1$. However, the c.o.v. (coefficient of variation) of the strength is about 2 times higher (0.11 instead of 0.06) for the open slit.

The diameter of the pin-dowel $d=6.7 \mathrm{~mm}$ (as for tests on wood) and the testing time: $5 \pm 2 \mathrm{~min}$.

The maximum permanent embedding deformation for the series $\mathrm{A}$ and $\mathrm{B}(b / d=10)$ was between 11 and $21 \mathrm{~mm}$ with a mean value of about $16 \mathrm{~mm}(=2.4 \cdot d)$. For series $\mathrm{C},(b / d=5)$, this was between 4 to $7 \mathrm{~mm}$ with a mean near $7 \mathrm{~mm}(1 d)$. For the series $\mathrm{B}$ and $\mathrm{C}$ with a half round slit, splitting occurred in the middle of the plate thickness and there were local shear lines of compression failure. For series A, with closed slit, there was only splitting in one specimen.

Because there is no difference of the (for density corrected) strengths of $\mathrm{A}$ and $\mathrm{B}$, the open half round slit can be used as test specimen.

It can be concluded that the embedding strength follows Eq. 27 with $c=1$, although the plastic deformation is limited (splitting effect) at small values of $b / d$. Not the empirical parameter of the investigation of Budianto et al. (1977): $a / d$ is determining for the strength, but $b / d$, where $b$ is the spreading width, showing that a theoretical explanation of empirical relations always is necessary.

The embedding strength of nailed particle boards to wood joints is only the same as in the embedding strength test for thick dowels in thin plates where the pin remains straight at failure and the embedding strength thus is (Eq. 9):

$\sigma_{s}=\sigma_{c} \cdot \sqrt{b / d}$.
Because the strength depends on the possible spreading, $b$ is equal to the nail row distance.

For the failure case of the pin by two plastic hinges in the pin, there only is a small effective bearing length $l$ of the nail of $l \approx(1$ to 1.5$) \cdot d$, near the edge of the plate. Now spreading is three dimensional, not only in b-direction but also in the a-direction, (the thickness direction) and the embedding strength is:

$\sigma_{s}=\sigma_{c b} \cdot \sqrt{a \cdot b /(l \cdot d)}$.

In this case $\sigma_{c b}$ is much higher than the compression strength because of the high strength of the boundary layers at the edge where the nail is bearing.

The bearing length 1 of the nail follows from:

$l=d \sqrt{\frac{\sigma_{e}}{\sigma_{h}} \cdot \frac{2 / 3}{1+\sigma_{s} / \sigma_{h}}}$

and the load on the nail is: $F=\sigma_{s} \cdot d \cdot l$ or:

$F=\sigma_{s} d^{2} \sqrt{\frac{\sigma_{e}}{\sigma_{h}} \cdot \frac{2 / 3}{1+\sigma_{s} / \sigma_{h}}}$,

where $\sigma_{s}$ is the embedding strength according to Eq. 31 and $\sigma_{e}$ is the ultimate bending flow stress of the nail and $\sigma_{h}$ is the embedding strength of wood in the particle board to wood connection. $\sigma_{s}$ can be found by iteration of " $l$ " according to Eq. 31 and Eq. 32. But also a closed solution and simplification is possible what will be discussed in the next article about nailed particle boards to wood joints.

\section{Conclusion}

- The quasi linear dependence of the embedding strength on the density can be explained. The 2 constants of the line have a constant ratio as explained by the theory.

- The high embedding strength is explained by confined dilatation due to the spreading effect as follows from the theory of plasticity.

- Splitting has no effect on spreading and therefore the embedding strength did not show an influence of the boundary conditions around the dowel (open or closed slit).

- Besides the plastic mechanism, a brittle splitting mechanism occurs at the dowel, explaining the volume effect for small dowels. Due to this splitting, flow is possible.

- Based on the spreading and the volume effect, the empirical equations of the extended investigation of Budianto et al. (1977) can exactly be explained by the theoretical expressions. For instance, Eq. 18 explains as well the straight part as the curved part of the line of Fig. 4 and Eq. 26 explains the change of the slope of the lines of Fig. 5. 
- The highest ultimate embedding strength is due to a local mechanism at the dowel as is verified in the TU-Delft investigation.

- The theory shows the embedding strength of Fig. 6 to be dependent on the $b / d$ ratio and not on the $a / d$ ratio of Fig. 3. This also follows from Dutch measurements at constant $a / d$ with different $b / a$ ratios. The verification of Eq. 9 or Eq. 27 follows from tests with one dowel diameter at different $b / d$ ratios. These tests are lacking in Budianto et al. (1977) and it is necessary to adapt the Codes at these points for the right design.

- The theory and the TU-Delft investigation did show a very high embedding strength for nails with a limited working length due to 3 -dimensional spreading. This will be shown in a following article.
Open Access This article is distributed under the terms of the Creative Commons Attribution Noncommercial License which permits any noncommercial use, distribution, and reproduction in any medium, provided the original author(s) and source are credited.

\section{References}

Budianto T, Ehlbeck J, Hemmer K, Herröder W, Lautenschläger R, Meickl G, Meyer K-H, Mistler H-L, Müller P, Rathfelder M, Roßbach S, Steck G, Wenz J (1977) Karlsruher Forschungsarbeiten und Versuche im Ingenieursholzbau von 1972 bis 1977. Bauen Holz 79(5):210-212. Figures 2 and 4 are copied with kind permission of Springer Science and Business Media

van der Put TACM (2006) Derivation of the bearing strength perpendicular to the grain of locally loaded timber blocks. In review, see: Publication Series 2006, nr. 3 on http://www.dwsf.nl/ downloads.htm, accessed 17 March 2008 